\title{
Knowledge and Practices Assessment of Decontamination during Root Canal Treatment in Saudi Arabia
}

\author{
Necdet Adanir ${ }^{1}$, Hassan Alkhalaf ${ }^{* 2}$, Maram H. Alanazi ${ }^{3}$, Asma S. Alghamdi ${ }^{4}$, Abdullah A. Aljami ${ }^{2}$, Amjad \\ O. Aljohani ${ }^{5}$, Khames T. Alzahrani ${ }^{6}$ \\ ${ }^{1}$ Department of Restorative Dentistry, College of Dentistry, King Faisal University, Al Ahsa, Saudi Arabia. \\ ${ }^{2}$ Restorative and Dental Materials Department, Undergraduate, King Faisal University, Saudi Arabia. \\ ${ }^{3}$ Restorative and Dental Materials Department, Intern, AlFarabi Dental College, Riyadh, Saudi Arabia. \\ 4Restorative and Dental Materials Department, Intern, King Abdulaziz University, Jeddah, Saudi Arabia. \\ ${ }^{5}$ Restorative Department and Dental Materials Department, AlFarabi Priavate Collage, Jeddah, Saudi Arabia. \\ ${ }^{6}$ Restorative and Dental Materials Department, BDS, PGD Endo, Ministry of Health, Saudi Arabia. \\ *Corresponding author: Hassan Alkhalaf; hymk-0101@ @otmail.com
}

Received 30 December 2020;

Accepted 06 January 2020;

Published 11 January 2021

\begin{abstract}
Introduction: Bacteria play a crucial role in the pathogenesis of pulpal and periradicular pathoses. For example: Apical periodontitis is an inflammation of dental periapical tissues developed as a response to colonization of microorganisms in root canal system. The elimination of microbial species from the infected root canal system necessitates a strict aseptic condition for Instrumentation, disinfection and inter appointment medication. Enterococcus faecalis and Candida albicans had been commonly associated organisms in treatment-resistant infections. Methods: This was an observational cross-sectional study based on web-survey questionnaires developed by the authors with high confidence. The sample size was 304 estimated using the Qualtrics calculator with a confidence level of $95 \%$ and a margin of error of 5\%. The questionnaire is divided into sections, regarding endodontic practice include endodontic education, years of experience, used of rubber dam or no and how to used irrigant solution whoever Sodium hypochlorite $(\mathrm{NaOCl})$, Chlorhexidine, chelating agents (EDTA) or (MTAD) and reasons of every irrigant that prevents during root canal treatment. Data were collected using Google forms and prepared for analysis using Microsoft Excel. Statistical analysis was performed using the SPSS software. Results: A total of 304 participants responded, $44.1 \%$ dental interns, 39.5\% General Dentist, 9.9\% Endodontist, $6.3 \%$ other dental specialty. the Probable reasons that prevent them using rubber dam during root canal treatment are: $5.9 \%$ due to difficulty to apply, $9.2 \%$ due to lack of materials, $5.3 \%$ because it is a time-consuming, $1.6 \%$ said that other isolation methods are enough (partial isolation), 3.9\% because of multiple reason combined together. $48.7 \%$ they always use rubber dam, so they did not choose any of the previous reasons. Conclusion: In conclusion, we identify and understand, most of the participants "always using rubber dam" during root canal treatment and the most common reasons was time consumption that prevented dentist from using rubber. In this study majority of participants preferred $\mathrm{NaOCl} 81.9 \%$ and $7.1 \%$ don't use it.
\end{abstract}

Keywords: Root Canal Treatment, Endodontic Difficulties, Cleaning and Shaping

\section{Introduction}

Root canal treatment (RCT) is a challenge because of ramifications and anatomic irregularities present in the root canal systems ${ }^{[1]}$. Residual microbes that have not been removed from the root canal system are the number one cause of post-treatment apical periodontitis for improperly managed root canal treatments (RCTs) ${ }^{[2]}$. During endodontic treatment, root canal instrumentation is essential, but not sufficient, in removing infected dentin from the root canal system. Therefore, root canal irrigants play an indispensable role in areas inaccessible to instruments, such as ramifications, anatomic irregularities, and dentine tubules ${ }^{[2,3]}$. One of the most common irrigants used in RCTs is sodium hypochlorite $(\mathrm{NaOCl}) . \mathrm{NaOCl}$ solution has many advantages, such as its ability to mechanically flush debris from the canal and dissolve vital and necrotic tissue, its antimicrobial action, and its lubricating action ${ }^{[4]}$. Sequential use of $17 \%$ ethylenediaminetetraacetic acid (EDTA) with $\mathrm{NaOCl}$ is an effective method in removing potentially contaminated smear layers ${ }^{[5]}$. Another commonly used irrigation solution, chlorhexidine (CHX), has a wide spectrum of 
antimicrobial activity, affords sustained action, and has little toxicity ${ }^{[6]}$. A $2 \%$ chlorhexidine rinse also enhances the rate of successful disinfection of the root canal system ${ }^{[6]}$. Besides, calcium hydroxide $\left(\mathrm{Ca}(\mathrm{OH})_{2}\right)$ is a widely used intracanal medicament for root canal disinfection in cases where root canals are infected ${ }^{[7]}$. The researchers concluded that $\mathrm{Ca}(\mathrm{OH})_{2}$ needs to be used in infected cases to greater predictably acquire disinfection ${ }^{[8]}$.

During root canal treatments, rubber dam isolation has been considered as a standard of care ${ }^{[9]}$. Previous studies have shown that general dentists do not use rubber dam isolation during RCTs despite its importance and necessity in the success of $\operatorname{RCTs}^{[10,11]}$.

Although it has been reported in many studies that decontamination and disinfection protocols are important in the success of endodontic treatments, a regional study that was done in Jeddah with 103 participants showed a limited use of chelating agents and intracanal medicaments during RCTs ${ }^{[12]}$. According to this study result, Albahiti ${ }^{[12]}$ also concluded that there is need to have a larger sample within Saudi Arabia to understand and investigate the challenges that dental practitioners may face. Furthermore, the reasons for lack of knowledge or failure to practice proper decontamination techniques during RCTs remain uncertain. Therefore, the aim of our study was to assess the knowledge of dental practitioners (interns, general dentists, and endodontists) about decontamination during RCTs and the practice of techniques used in the private and government sectors of different regions (Western, Central, and Eastern) in Saudi Arabia, with further investigation of the reasons and challenges for lack of knowledge of or failure to practice proper decontamination techniques for RCTs. In this study, we also set out to determine the significant procedural difficulties and challenges encountered by undergraduates during endodontic RCTs. When looking at prior research, we found a few samples that explored the same ideas, but nothing similar was found for Saudi Arabia. Our secondary objective was to assess dental undergraduates' perceptions about the difficulties they face while performing RCTs in Saudi Arabia and then inspect the students' and interns' responses to these difficulties and make recommendations to educators about possible resolutions.

\section{Materials and Methods}

This cross-sectional questionnaire study took place in the period July 2020-November 2020 and has been used to assess the knowledge and practice related to decontamination during root canal procedures and cross-infections during endodontic procedures in the government and private sectors in Saudi Arabia, specifically Al Riyadh, Al Ahsa, Al Khobar, Dammam, and Jeddah. The sample size was estimated using the Qualtrics calculator with a confidence level of $95 \%$; the sample size was 304 . The participants' responses to the questions were collected electronically using online Google forms, and completed hard copies were also collected from the clinics.

A structured questionnaire was used as the study tool. This tool was developed after reviewing related studies carried out in Saudi Arabia and elsewhere. The first section collected sociodemographic data (gender, nationality, degree of education, type of sector, years of practice, city of work, and school of education). The second section collected data regarding endodontic practice, including endodontic education, years of experience, whether rubber dams were used, and irrigant solution use considering sodium hypochlorite $(\mathrm{NaOCl})$, chlorhexidine, and chelating agents (EDTA) or (MTAD), and the reasons that prevented their use of irrigants during RCTs. We also investigated the reasons that prevented the intracanal use of calcium hydroxide $\left(\mathrm{Ca}(\mathrm{OH})_{2}\right)$ during root canals.

We included male and female participants who were undergraduate students, general dentists, endodontists, and other dental specialists who had finished pre-clinical endodontic courses and agreed to participate. Included students had previously attended and finished a preclinical endodontics course and had entered the clinical endodontic program. Students who had not completed a pre-clinical course in endodontics were excluded from the study.

\section{Statistical analysis}

The data were collected by computer using Microsoft Office's Excel software program (2019) for Microsoft Windows. Data were then transferred to the Statistical Package of Social Science Software (SPSS) program, version 20 (IBM SPSS Statistics for Windows, Version 20.0. Armonk, NY: IBM Corp).

\section{Results}

Of the 304 participants, $44.1 \%$ were dental interns, $39.5 \%$ were general dentists, $9.9 \%$ were endodontists, and $6.3 \%$ were from other dental specialties. Of all participants, $49.7 \%$ worked in the government sector, and $50.3 \%$ worked in the private sector. Furthermore, $17.8 \%$ were dentists working in government hospitals, 9.9\% worked in primary healthcare centers, $41.8 \%$ worked in university hospitals, and $30.3 \%$ did not work in the government sector at all.

The participants were from many Saudi cities: $42.8 \%$ were from Riyadh, $8.9 \%$ were from Jeddah, $3.6 \%$ were from AlMadinah, $16.4 \%$ were from Al-Ahsa, $1.3 \%$ were from Hail, $2 \%$ were from Abha, $10.2 \%$ were from Dammam, $1 \%$ were from Qassim, 3.6\% were from Al-Khobar, $0.3 \%$ were from Khamis Moshait, $0.3 \%$ were from Arrass, $8.2 \%$ were from Jazan, $0.3 \%$ were from Albaha, $0.3 \%$ were from Kharje, and $0.3 \%$ were from Makkah.

Most of the participants were Saudi Arabian (80.9\%), and the rest were of other nationalities. Gender was almost evenly split among the participants: $48.4 \%$ female, $51.6 \%$ male. Our sample had participants with a range of practical experience: $43.8 \%$ had less than 1 year of practice, $32.9 \%$ had less than 5 years of practice, $13.5 \%$ had 5-10 years of practice, and 9.5\% had more than 10 years of practice. Of the participants, $57.2 \%$ had graduated from public schools, and $42.4 \%$ had graduated from private schools; $15.5 \%$ had a PhD or MSc certificate in endodontics, $40.8 \%$ had completed a short program (such as a short-term course or a workshop in endodontics), and $43.4 \%$ had no additional endodontic education. Fifty percent of the participants had less than one qualification year, $30.9 \%$ had fewer than five qualification years, $12.8 \%$ had less than ten qualification years, and $5.9 \%$ had more than ten qualification years. Of our participants, $76.3 \%$ always used rubber dams during RCTs, $17.8 \%$ sometimes used rubber dams, and $5.6 \%$ never used rubber dams.

When asked for the reasons that prevented them from using rubber dams during RCTs, $5.9 \%$ cited difficulties in application, 9.2\% cited lack of materials, 5.3\% said it was time consuming, $1.6 \%$ said that other isolation methods were sufficient (partial isolation), and $3.9 \%$ cited a combination of reasons. Of the respondents, $48.7 \%$ said they always used rubber dams, so they did not select any of the aforementioned reasons. Furthermore, 25.7\% only used $\mathrm{NaOCl}$ as an irrigant solution, $0.7 \%$ only used chlorhexidine, $1.3 \%$ used saline, and the remainder used multiple 
types of irrigant solutions $(\mathrm{NaOCl}, \mathrm{CHX}$, hydrogen peroxide $\left(\mathrm{H}_{2} \mathrm{O}_{2}\right)$, or EDTA), depending on what was readily available. Sodium hypochlorite $(\mathrm{NaOCl})$ was used as the sole irrigant by $81.9 \%$ of respondents, while $7.1 \%$ did not use $\mathrm{NaOCl}$ because they considered mechanical measures adequate, and the remainder of responses cited a lack of materials, the time-consuming nature of use, and opinions about the effectiveness of saline alone and the superfluous nature of dams as reasons for their lack of use. Of the respondents, $8.6 \%$ always used chlorhexidine as an adjunct irrigant to sodium hypochlorite $(\mathrm{NaOCl})$ for every canal procedure, $58.9 \%$ never used an adjunct irrigant with $(\mathrm{NaOCl})$, and the majority did not use it for two reasons: $36 \%$ said "there is no need for using it", and $24 \%$ said "using mechanical measures is adequate". Furthermore, $48 \%$ of the participants sometimes used chelating agents, such as EDTA or MTAD, during RCTs, and 37\% always used them. Another $51.3 \%$ did not use chelating agents during endodontic treatments because they were unaware of their effect. For intracanal medication, 23\% always used calcium hydroxide $\left(\mathrm{Ca}(\mathrm{OH})_{2}\right)$, and $64 \%$ used it occasionally. The rest of the respondents did not use it at all because $35 \%$ were unaware of its effect; $16 \%$ did not use it for one of two reasons: they were unaware of its effect, or the material was not available in their workplace.

Out of the 134 dental interns, 127 used rubber dams during endodontic treatments, and the remainder used them occasionally. Out of the 120 general dentists surveyed, 71 used rubber dams during endodontic treatments, and 37 occasionally used them, while 12 did not use them at all. Of the 30 endodontists, 25 used rubber dams during endodontic treatments, 3 occasionally used them, and 2 did not use them. Only 9 out of the 19 other specialists always used rubber dams, 7 occasionally used them, and 3 never used them.

The majority of respondents with degrees (249 out of 304) used $\mathrm{NaOCl}$ for every canal procedure, 46 sometimes used it and 7 never used it; 28 out of the 30 endodontists, 108 out of the 134 dental interns, 98 out of the 120 general dentists, and 15 out of 19 used $\mathrm{NaOCl}$ for every canal procedure. Thirteen out of the 40 dental interns who did not use $\mathrm{NaOCl}$ considered mechanical measures adequate, and the remainder selected the response "Normal saline is effective instead of $\mathrm{NaOCl}$ ". Thirteen out of the 42 general dentists who did not use $\mathrm{NaOCl}$ said that using mechanical measures was adequate and there was no need to use $\mathrm{NaOCl}$. Only one endodontist did not use $\mathrm{NaOCl}$ because of a lack of materials. Thirty out 134 dental interns used $\mathrm{NaOCl}$ as an irrigant solution, 28 out of 134 used $\mathrm{NaOCl}$ and saline solution together, and 31 used $\mathrm{NaOCl}$ and saline solution and EDTA as canal irrigants. Thirty-one out 120 general dentists used $\mathrm{NaOCl}$ as an irrigant solution, 30 used $\mathrm{NaOCl}$ and saline solution, and 14 used $\mathrm{NaOCl}$ and saline solution and EDTA as canal irrigants. Ten out of 30 endodontists used $\mathrm{NaOCl}$ as an intracanal irrigant solution, and the rest used chlorhexidine, EDTA, $\mathrm{H}_{2} \mathrm{O}_{2}$, and combinations of these.

Most dental interns (82 out of 134) did not use chlorhexidine as an adjunct irrigant to sodium hypochlorite, 41 of them sometimes used it, and only 11 always used it. Seventy-one out of 120 general dentists and 13 out of 30 endodontists never used chlorhexidine as an adjunct irrigant to sodium hypochlorite, 37 and 15, respectively, sometimes using it and 12, 2 always. The majority of dental interns did not use chlorohexidine because they considered mechanical measures adequate: 57 out of 134 saw no need for its use, and 34 considered using mechanical measures adequate. Forty out of 120 general dentists saw no need for the use of chlorhexidine, and 28 believed that mechanical measures were adequate. Six of the endodontists surveyed considered mechanical measures alone to be adequate, eight saw no need to use chlorhexidine, and four endodontists said that saline was effective. Sixty-one out of 134 dental interns used the chelating agents EDTA or MTAD occasionally, and 54 always used them. While 65 of the general dentists occasionally used chelating agents and 37 always used them, 18 out of the 30 endodontists always used them, and 11 occasionally used them. The most common reason that dental interns, general dentists, and endodontists cited for not using chelating agents (EDTA or MTAD) was a lack of materials. The vast majority of dental interns used intracanal medication (calcium hydroxide) occasionally (85 out of 134), and 34 always used it. This is comparable to the responses from the general dentists: 83 out of 120 occasionally used calcium hydroxide, 22 always used it. Sixteen out of 30 endodontists used calcium hydroxide occasionally, and 11 always used it. Moreover, 109 out of 304 participants did not use calcium hydroxide as an intracanal medication because they were unaware of its effects or cited a combination of lack of materials, the time-consuming nature of use or that there was no need for using it. Finally, we noticed that there was no significant difference between the materials used and the reasons cited between the private and government sectors, so dentists or specialists seem to be deciding which materials will be used and why they should be used.

\section{Discussion}

This country-wide cross-sectional study aimed to poll dental practitioners to evaluate their knowledge and practices related to the use of decontamination materials during RCTs. The survey was distributed in the main cities of Saudi Arabia to dental practitioners (interns, GPs, and endodontists) and included questions about decontamination materials. The first part of the questionnaire included the background, and the second part of the questionnaire included general questions about each material, whether it was used, why it was or was not used, and detailed questions about certain decontamination materials to assess their knowledge. The main objectives addressed the knowledge and practice of the five most common decontamination materials used on a daily basis in endodontic treatments. Each of the mentioned variables in the questionnaire was either found separately addressed in prior research or had few participants in Saudi Arabia ${ }^{[9,12,13]}$. Therefore, this research aimed to make a thorough evaluation of all common decontamination materials for the same kinds of participants with a larger sample size and include more of the country's important regions. As a secondary objective, we intended to compare participants and discover whether a lack of proper practice or knowledge or both were dominating by analyzing the answered questionnaire; our analysis included their degree of education, workplace, city of residence, nationality, gender, and years of dentistry practice. The study's methodology focused on evaluating all common decontamination materials used in endodontic treatments that were also discussed in a study, ${ }^{[12]}$ which was a study conducted in Jeddah, while the current study also included the main regions of the country (East, Middle, and West).

In this study, $76.3 \%$ of participants reported that they always used rubber dams during RCTs, $17.8 \%$ sometimes used them, 5.6\% did not use them at all, and $1.6 \%$ used other isolation techniques (partial isolation). Rubber dams were mostly used by dental interns and endodontists $(94.7 \%$ and $83.3 \%$, respectively) and were least used by general dentists $(59.1 \%)$. The results of this study are partially consistent with a study result reported by Madarati ${ }^{[9]}$. Although it increased compared to the result of a study 
reported by Al-Fouzan, ${ }^{[13]}$ this study showed that the use of rubber dam was the lowest as an isolation technique among general dentists. The reasons for not using rubber dam isolation varied; common responses were a lack of materials $(9.2 \%)$, difficulty in the application $(5.9 \%)$, and the time-consuming nature of use (5.3\%). The second common reason that prevented dentists from using rubber dams was the time required for use is consistent with a published study conducted by Whitworth et al., ${ }^{[14]}$ while the unavailability of materials was cited as the main barrier to use by $40.5 \%$ of participants in this study. Many studies have evaluated isolation elsewhere in the world, such as Lithuania, ${ }^{[15]}$ where in a group of 1431 general practitioners $66 \%$ of participants did not use rubber dams for isolation, or in other study in Turkey, ${ }^{[16]}$ where out of 602 dentists less than $2 \%$ reported the use of rubber dams.

As part of the main objective of this research, questions about the types of irrigants preferred by dentists in endodontic treatments $\left(\mathrm{NaOCl}, \mathrm{CHX}\right.$, EDTA, and $\left.\mathrm{H}_{2} \mathrm{O}_{2}\right)$ were included in the questionnaire. Sodium hypochlorite, chlorhexidine, chelating agents (EDTA, MTAD), and $\mathrm{Ca}(\mathrm{OH})_{2}$ as an intracanal medicament were specifically investigated in the questionnaire to determine whether they were used and the reasons that prevented the use of these materials. Irrigating canals during root canal treatment has proven to be effective in many aspects, and a decrease in bacterial colonies has been shown after the use of $\mathrm{NaOCl}{ }^{[17,18]}$. The eradication of bacteria is not the only concern; organic materials that can prevent the complete decontamination of the root canal and can only be flushed and dissolved by using irrigants such as $\mathrm{NaOCl}$ are also of concern ${ }^{[19]}$. In this study, the majority of participants preferred $\mathrm{NaOCl}(81.9 \%)$, worryingly a small proportion of participants $(7.1 \%)$ did not use it because they believed that mechanical measures were adequate. This result of this study was in accordance with other studies ${ }^{[12,16]}$. Further, $8.6 \%$ of the participants always used CHX irrigant as an adjunct irrigant to sodium hypochlorite $(\mathrm{NaOCl})$, while $58.9 \%$ never used an adjunct irrigant with $(\mathrm{NaOCl})$. The majority did not use it for two reasons: $36 \%$ said "there is no need for using it", $24 \%$ said "using mechanical measures is adequate". In this study, CHX was not used as an irrigant as often as compared to the findings of Kaptan et al. ${ }^{[16]}$ : only $4.6 \%$ of dentists used it. In their study, Kaptan et al. ${ }^{[16]}$ found $43.9 \%$ of dentists with 16-20 years of experience used chlorhexidine, and it was used least in the group with $0-5$ years of experience.

The results of this study showed that $23 \%$ of participants reported that they always used calcium hydroxide, and $64 \%$ occasionally used it, while the remainder did not use it at all because $35 \%$ were unaware of its effect. Comparable results to those in a study were observed for participants who reported using $\mathrm{Ca}(\mathrm{OH})_{2}$ : always $29.8 \%$, sometimes $69 \%$, and never $1.2 \%{ }^{[12]}$.

Chelating agents, such as EDTA, are used as the final irrigant before obturation to ensure that the smear layer is removed $^{[3]}$. In this study, while $40.2 \%$ of dental interns used the chelating agents EDTA or MTAD always, $30.8 \%$ of the general dentists used chelating agents. However, 18 out of the $30(60 \%)$ endodontists always used them, and 11 (27.2\%) occasionally used them. Compared to a questionnaire study of Albahiti, ${ }^{[12]}$ the proportion of always use of chelating agents increased in this study.

This study's findings will aid in a better understanding of the differences in knowledge among Saudi dental practitioners in both the government and private sectors. It also provides insight into how much of that knowledge is applied during their routine endodontic treatments and looks for the reasons that prevent dentists from using these materials. This study included a larger sample size and involved more cities than previously published papers in the country. The limitation of this study involved the sample size and the unequal distribution of the questionnaire in each city and among dentists in different specialties and at different levels of education. Further investigations in Saudi Arabia are needed with more participants and increased distribution in more cities to obtain more data on the endodontic practices and knowledge of dentists across the country.

\section{Conclusion}

The present study was conducted to assess the knowledge of dental practitioners (interns, general dentists, and endodontists) about decontamination during RCTs and the practiced techniques used in private and government sectors in different regions (Western, Central, and Eastern) in Saudi Arabia. We also investigated the reasons for and challenges of either the lack of knowledge or the lack of practicing of proper decontamination techniques during RCTs. In conclusion, most of the participants reported that they always used a rubber dam during RCTs, and the most common reason for not using a rubber dam was the time-consuming nature of its use. In this study, the majority of participants preferred $\mathrm{NaOCl}(81.9 \%)$, while $7.1 \%$ did not use it. Also, it has been observed that the proportion of always using chelating agents is increasing.

\section{Conflicts of Interest}

The authors have no conflicts of interest to declare.

\section{Source of Funding}

This study did not receive any specific grant from funding agencies in the public, commercial, or not-for-profit sectors.

\section{Ethical approval}

This study was approved by the institutional review board at King Faisal University, Saudi Arabia, (reference: KFU-REC/2020-1104)

\section{Reference}

[1] Vertucci FJ. Root canal anatomy of the human permanent teeth. Oral Surg Oral Med Oral Pathol. 1984;58(5):589-599.

[2] Kováč J, Kováč D. Microbial decontamination of the root canals of devitalized teeth. Epidemiol Mikrobiol Imunol. 2012;61(4):87-97.

[3] Ok E, Adanir N, Ozturk T. Antibacterial and smear layer removal capability of oregano extract solution. Eur $\mathrm{J}$ Dent. 2015;9(1):20-24.

[4] Johnson WT, Noblett WC, Cleaning and Shaping. In: Torabinejad M and Walton RE, Endodontics: Principles and Practice. 4th ed., St. Louis: Saunders-Elsevier; 2009:p263

[5] Cobankara FK, Adanr N, Belli S. Evaluation of the influence of smear layer on the apical and coronal sealing ability of two sealers. J Endod. 2004;30(6):406-409.

[6] Zamany A, Safavi K, Spångberg LS. The effect of chlorhexidine as an endodontic disinfectant. Oral Surg Oral Med Oral Pathol Oral Radiol Endod. 2003;96(5):578-581. 
[7] Law A, Messer H. An evidence-based analysis of the antibacterial effectiveness of intracanal medicaments. J Endod. 2004;30(10):689-694.

[8] Chan W, Chowdhury NR, Sharma G, Zilm P, RossiFedele G. Comparison of the biocidal efficacy of sodium dichloroisocyanurate and calcium hydroxide as intracanal medicaments over a 7-day contact time: An ex vivo study. J Endod. 2020;46(9):1273-1278.

[9] Madarati AA. Why dentists don't use rubber dam during endodontics and how to promote its usage? BMC Oral Health. 2016;16:24.

[10] Anabtawi MF, Gilbert GH, Bauer MR, et al. Rubber dam use during root canal treatment: findings from The Dental Practice-Based Research Network. J Am Dent Assoc. 2013;144(2):179-186.

[11] Lawson NC, Gilbert GH, Funkhouser E, et al. General dentists' use of isolation techniques during root canal treatment: from the National Dental Practice-based Research Network. J Endod. 2015;41(8):1219-1225.

[12] Albahiti MH. Knowledge and practices of decontamination during root canal treatment by dentists in Jeddah. Saudi Dent J. 2020;32(4):213-218.
[13] Al-Fouzan KS. A survey of root canal treatment of molar teeth by general dental practitioners in private practice in Saudi Arabia. Saudi Dent J. 2010;22(3):113-117.

[14] Whitworth JM, Seccombe GV, Shoker K, Steele JG. Use of rubber dam and irrigant selection in UK general dental practice. Int Endod J. 2000;33(5):435-441.

[15] Peciuliene V, Rimkuviene J, Aleksejuniene J, Haapasalo M, Drukteinis S, Maneliene R. Technical aspects of endodontic treatment procedures among Lithuanian general dental practitioners. Stomatologija. 2010;12(2):42-50.

[16] Kaptan RF, Haznedaroglu F, Kayahan MB, Basturk FB. An investigation of current endodontic practice in Turkey. ScientificWorldJournal. 2012;2012:565413.

[17] Siqueira JF Jr, Batista MM, Fraga RC, et al. Antibacterial effects of endodontic irrigants on black-pigmented gramnegative anaerobes and facultative bacteria.J Endod 1998;24:414-416.

[18] Rocas IN, Siqueira JF Jr. Comparison of the in vivo antimicrobial effectiveness of sodium hypochlorite and chlorhexidine used as root canal irrigants: a molecular microbiology study. J Endod 2011;37:143-50.

[19] Haapasalo M, Shen Y, Wang Z, Gao Y. Irrigation in endodontics. Br Dent J. 2014;216(6):299-303. 